\title{
Mortality estimates among adult patients with severe acute respiratory infections from two sentinel hospitals in southern Arizona, United States, 2010-2014
}

Steve R. Barnes ${ }^{1,2}$, Zimy Wansaula ${ }^{1}$, Kristen Herrick ${ }^{3}$, Eyal Oren², Kacey Ernst ${ }^{2}$, Sonja J. Olsen ${ }^{4}$ and Mariana G. Casal ${ }^{1 *}$ (D)

\begin{abstract}
Background: From October 2010 through February 2016, Arizona conducted surveillance for severe acute respiratory infections (SARI) among adults hospitalized in the Arizona-Mexico border region. There are few accurate mortality estimates in SARI patients, particularly in adults $\geq 65$ years old.

The purpose of this study was to generate mortality estimates among SARI patients that include deaths occurring shortly after hospital discharge and identify risk factors for mortality.

Methods: Patients admitted to two sentinel hospitals between 2010 and 2014 who met the SARI case definition were enrolled. Demographic data were used to link SARI patients to Arizona death certificates. Mortality within 30 days after the date of admission was calculated and risk factors were identified using logistic regression models.

Results: Among 258 SARI patients, 47\% were females, 51\% were white, non-Hispanic and 39\% were Hispanic. The median age was 63 years (range, 19 to 97 years) and 80\% had one or more pre-existing health condition; 9\% died in hospital. Mortality increased to 12\% (30/258,30\% increase) when electronic vital records and a 30-day posthospitalization time frame were used. Being age $\geq 65$ years ( $\mathrm{OR}=4.0 ; 95 \% \mathrm{Cl}: 1.6-9.9)$ and having an intensive care unit admission ( $\mathrm{OR}=7.4 ; 95 \% \mathrm{Cl}$ : 3.0-17.9) were independently associated with mortality.
\end{abstract}

Conclusion: The use of electronic vital records increased SARI-associated mortality estimates by $30 \%$. These findings may help guide prevention and treatment measures, particularly in high-risk persons in this highly fluid border population.

Keywords: Arizona, Death certificates, Fatal outcome, Influenza, Respiratory tract diseases, Surveillance

\section{Background}

Pneumonia and influenza remain leading causes of morbidity and mortality in the United States [1]. Adults aged 65 years and older, children under five years, pregnant women and those with chronic medical conditions such as asthma or heart disease are at high risk for complications that might lead to hospitalization and death [2-5]. Each year, influenza causes an estimated 140,000710,000 hospitalizations and 12,000-56,000 deaths in

\footnotetext{
* Correspondence: Mariana.Casal@azdhs.gov

${ }^{1}$ Arizona Department of Health Services, Border Infectious Disease

Surveillance Program, 400 West Congress, Suite 116, Tucson, AZ 85701, USA

Full list of author information is available at the end of the article
}

the United States alone [6]. In 2015, the pneumonia and influenza mortality rate was estimated at $18 / 100,000$ among U.S. residents of all ages and 102/100,000 in those aged $\geq 65$ years [7]. In 2015, among Arizona residents, the pneumonia and influenza mortality rate was estimated at 11/100,000 and 58/100,000 for all age groups and adults aged $\geq 65$ years, respectively $[7,8]$.

The 2009 H1N1 influenza pandemic highlighted the need to better understand severe influenza in persons admitted to a hospital. In response, the World Health Organization (WHO) recommended enhanced influenza surveillance guidelines and implementation of surveillance for severe acute respiratory infections (SARI) in member 
countries [9, 10]. The Arizona Department of Health Services (ADHS) has conducted statewide influenza surveillance since 1997. Surveillance activities include monitoring influenza-like illness (ILI) among ambulatory patients, tracking laboratory-confirmed cases, monitoring ILI in sentinel schools, and testing and subtyping influenza viruses in specimens submitted to the Arizona State Public Health Laboratory. In 2010, in response to the WHO guidance, ADHS began conducting sentinel surveillance for SARI as part of the Centers for Disease Control and Prevention (CDC) Border Infectious Disease Surveillance program (BIDS) with the aim of describing the patterns of severe disease in a highly fluid border region. SARI surveillance was established in Pima County, Arizona along the U.S.-Mexico Border, and aimed to complement existing statewide influenza surveillance activities. Previous estimates of mortality among SARI patients in this population have been limited to in-hospital mortality, thus, deaths that occurred after hospital discharge were not captured [11]. The main objective of this analysis was to quantify mortality among adult patients hospitalized with SARI by capturing deaths occurring both in hospital and shortly after hospital discharge, and to identify potential risk factors for mortality.

\section{Methods}

This analysis was exempt from ethical approval because ADHS' Human Subjects Review Board determined that SARI surveillance was part of public health practice.

\section{Study sites}

Between October 2010 and February 2016, SARI surveillance was conducted at three sentinel sites in Pima County that routinely receive acutely ill patients and transferred patients from smaller hospitals in the border region. Selection of study sites has been described in detail elsewhere [11]. In brief, the ADHS, BIDS program identified hospitals in the four Arizona counties (Cochise, Pima, Santa Cruz, and Yuma) that border Mexico and receive mobile, foreign born and migrant populations. There were 11 acute care hospitals within this area; surveillance was initiated at five hospitals in 2010. Two of the participating hospitals had fewer than five participants in the first season, leaving three in the surveillance system. One of the three sentinel surveillance sites did not have data from electronic records accessible; therefore, only two sites were included in this mortality analysis. This analysis was limited to SARI patients who presented to the inpatient ward of St. Mary's Hospital or St. Joseph's Hospital in Pima County, Arizona. These two large acute care sites transfer pediatric cases to other regional hospitals; therefore, only adults $\geq 18$ years were included in this analysis. For this analysis, we defined each influenza season as beginning in week 40 and ending in week 39 of the consecutive year.

\section{Case ascertainment and data collection methods}

The details of the SARI surveillance methods have been described in detail elsewhere [11]. In brief, clinical teams at each surveillance site were trained on the SARI case definition (Additional file 1: Table S1) and surveillance procedures. Patients who presented at the emergency ward who were identified by the clinical team as meeting the SARI case definition were asked for verbal consent to participate. Data for enrolled patients were collected using a standardized case report form. Data collected included age, sex, race/ethnicity, residence, symptom onset date, admission date, existing underlying medical conditions, clinical suspicion of pneumonia (yes/no), chest radiograph findings (new abnormality, normal or not done), laboratory results (e.g., blood culture), and intensive care unit (ICU) admission. Due to infrequent ordering of bacterial cultures and recording of chest radiograph findings on case report forms, these variables were not included in the analysis. Nasopharyngeal swabs were collected from each SARI patient within $24 \mathrm{~h}$ of admission and tested for viral respiratory pathogens. Testing was performed using multiplex polymerase chain reaction (PCR) using the ResPlex II assay (v. 2.0) (Qiagen, Hilden, Germany) from 2010 to 2012 and the GenMark Respiratory Viral Panel assay from 2013 to 2014 (GenMark Diagnostics Inc., Carlsbad, CA, United States) [12, 13]. Viral targets included influenza viruses A and $\mathrm{B}$, human metapneumovirus (HMPV), parainfluenza viruses (1-4), respiratory syncytial virus (RSV) A and B, rhinoviruses, coronaviruses (229E, OC43, NL63, HKU1) and adenoviruses.

We conducted a retrospective review of electronic medical records of each SARI case enrolled between week 40, 2010 and week 39, 2014 to obtain name, date of birth, discharge date and discharge status (dead/alive). On March 3, 2015, we queried the State of Arizona Office of Vital Records database to find matches between SARI patients and electronic death certificates using patient name, sex and date of birth. Variables obtained from death certificates and used in this analysis included date of death, underlying cause, contributing cause(s), place of death (inpatient, outpatient, hospice, decedents residence or other) and manner of death (natural or unnatural). Patients with death certificates listing a date of death beyond the date of hospital discharge were assumed to have died outside the sentinel hospital and were verified by place of death listed on the death certificate. Patients who died of unnatural causes were excluded from mortality calculations.

\section{Statistical analysis}

Descriptive statistics of all SARI cases are reported as median values for continuous variables and frequencies 
and percentages for categorical variables. Mortality status was defined at 30-days post admission. The 30-day allcause mortality from hospital admission date was calculated and reported as a percentage and 95\% confidence interval. Deaths were categorized using International Classification of Disease, Tenth Revision (ICD-10) codes listed on death certificates for underlying and/or contributing cause of death. Cause of death was classified as pneumonia and influenza (ICD-10 codes J09-J18), respiratory and circulatory (ICD-10 codes J00-J99, I00-I99) or all-cause (all ICD-10 codes). Use of these categorizations for attributing cause of death due to influenza or other viral respiratory pathogens has been described elsewhere [14].

Predictors of mortality were identified by comparing patients who died with those who were alive at the 30day post admission time point, using bivariate and multivariate logistic regression analysis. In hospitalized patients with pneumonia, a comparable condition to SARI, one study found that deaths within 30 days of admission were generally directly related to pneumonia, whereas deaths beyond 30 days were unrelated to pneumonia [15]. Additionally, the Centers for Medicare \& Medicaid Services reported all-cause 30-day post admission mortality for patients with a principal diagnosis of pneumonia [16]. Therefore, we selected the 30-day post admission timeframe to capture SARI-related deaths while allowing our findings to be comparable with other research studies. Potential predictors examined in bivariate analysis included age, sex, race/ethnicity, length of hospital stay, time from symptom onset to hospital admission, ICU admission, presence of comorbidities $(\geq 1$, $\geq 2$ and $\geq 3$ comorbidities), individual comorbidities (hypertension, cardiac disease, lung disease, obesity, immunodeficiency and neurologic disorder), and the presence of individual viral pathogens (influenza viruses, parainfluenza viruses, coronaviruses, HMPV, RSV and rhinoviruses). Race/ethnicity was defined as a categorical variable with multiple levels. All other variables were defined as the presence or absence of the attribute. Independent predictors of mortality identified with a significance of $p<0.1$ in bivariate analysis were included in the multivariate models. Non-significant factors in multivariate models were dropped using stepwise backward selection.

Kaplan-Meier curves for 30-day mortality were generated to display patient survival by independent predictors of mortality, and curves were compared using the log-rank test. Patients were censored at death or 30 days post hospital admission. Complete follow-up data were available for all patients at 30 days post hospital admission. All analyses were performed using SAS software version 9.3 and statistical significance was set at an alpha level of 0.05 (SAS Institute, Inc., Cary, NC).

\section{Results}

A total of 306 SARI patients were admitted and enrolled by the two surveillance sites between week 40, 2010 and week 39, 2014; 48 did not meet the case definition and were excluded from further analyses leaving a total of 258 patients included in this analysis. Median age at admission was 63 years (range, 19-97 years) and 121 (47\%) were women. One hundred thirty-one (51\%) were white, non-Hispanic and 101 (39\%) were Hispanic. The median time from symptom onset to hospital admission was four days (interquartile range (IQR); 26 days), the median duration of hospital stay was five days (IQR, 3-9 days); 96 (37\%) patients were admitted to the ICU (Table 1).

Overall, 207 (80\%) patients had one or more underlying medical condition (27\% had one, $23 \%$ had two, $18 \%$ had three and $11 \%$ had four or more). The most common underlying medical condition was hypertension $(48 \%, n=125)$ followed by chronic lung disease (33\%, $n=84)$ and cardiovascular disease $(26 \%, n=69)$.

Nasopharyngeal specimens were collected and tested for 253 (98\%) patients. Of the samples tested, 113 (45\%) were positive for a viral respiratory pathogen. Nineteen percent of all the SARI samples and $42 \%$ of the positive samples $(n=47)$ had an influenza virus identified, $5 \%$ of the SARI and $12 \%$ of the positives $(n=13)$ had a parainfluenza virus, $8 \%$ of the SARI and $18 \%$ of the positives $(n=20)$ had a HMPV, $6 \%$ of the SARI and $12 \%$ of the positives $(n=14)$ had a coronavirus, and $3 \%$ of the SARI and $7 \%$ of the positives $(n=8)$ had a RSV (Table 1$)$.

\section{Mortality}

For all-cause mortality, 30/258 (12\%, 95\% CI 8-15\%) patients died within 30 days of hospital admission [23/30 died in-hospital (77\%)]. Among patients $\geq 65$ years, 22/ 135 (16\%, 95\% CI 11-24\%) died within 30 days of hospital admission [15/22 died in-hospital (68\%)]. Among those who died, the median age at admission was 72 years (range: $35-88$ years), the median duration of hospital stay was 6 days (range: $0-28$ days), and the median time from symptom onset to death was 12 days (range: 4-32 days). Twenty-two of thirty (73\%, 95\% CI 58-89\%) patients that died were admitted to the ICU [20/22 died in-hospital (90\%)] and 27/30 (90\%) patients had at least one underlying disease. An influenza virus was detected among 4/30 (13\%) patients who died. Allcause mortality by influenza season was similar, with 5 / 36 deaths (14\%) in the $2010-2011$ season, $3 / 23$ (13\%) in 2011-2012, 6/44 (14\%) in 2012-2013 and 16/125 (13\%) in 2013-2014.

Underlying and contributing cause of death codes were listed on death certificates for 28 (93\%) patients who died. Fourteen (50\%) were classified as underlying pneumonia and influenza deaths and $14(50 \%)$ as 
Table 1 Characteristics of all SARI patients, and by status (alive/dead)

\begin{tabular}{|c|c|c|c|c|}
\hline & $\begin{array}{l}\text { All patients } \\
n=258\end{array}$ & $\begin{array}{l}\text { Alive } \\
n=228\end{array}$ & $\begin{array}{l}\text { Dead } \\
n=30\end{array}$ & $p$ value \\
\hline Demographic Characteristics & $n(\%)$ & $n(\%)$ & $n(\%)$ & \\
\hline Age (median, range) & $63(19-97)$ & $62(19-97)$ & $72(35-88)$ & 0.006 \\
\hline Age $\geq 65$ years & $135(52 \%)$ & $113(50 \%)$ & $22(73 \%)$ & 0.018 \\
\hline Sex (female) & $121(47 \%)$ & $108(47 \%)$ & $13(43 \%)$ & 0.677 \\
\hline Race/Ethnicity & & & & 0.874 \\
\hline Asian & $4(2 \%)$ & $3(1 \%)$ & $1(3 \%)$ & \\
\hline African American & $6(2 \%)$ & $5(3 \%)$ & $1(3 \%)$ & \\
\hline Hispanic & 101 (39\%) & $89(40 \%)$ & $12(40 \%)$ & \\
\hline Native American & $16(6 \%)$ & $15(7 \%)$ & $1(3 \%)$ & \\
\hline White, non-Hispanic & $131(51 \%)$ & $116(49 \%)$ & $15(50 \%)$ & \\
\hline \multicolumn{5}{|l|}{ Clinical Characteristics } \\
\hline Duration of hospital stay (median; range) & $5(0-57)$ & $5(0-57)$ & $6(0-28)$ & 0.482 \\
\hline Days to seek care (median; range) & $4(0-10)$ & $4(0-10)$ & $3(1-7)$ & 0.288 \\
\hline Admission to intensive care unit & $96(37 \%)$ & $74(32 \%)$ & $22(73 \%)$ & $<.0001$ \\
\hline Clinical suspicion of pneumonia ${ }^{a}$ & $184(71 \%)$ & $159(70 \%)$ & $25(83 \%)$ & 0.175 \\
\hline$\geq 3$ Comorbidities & 75 (29\%) & $64(28 \%)$ & $11(36 \%)$ & 0.332 \\
\hline$\geq 1$ Comorbidity & 207 (80\%) & $180(78 \%)$ & 27 (90\%) & 0.165 \\
\hline Cardiac disease & $69(26 \%)$ & $61(26 \%)$ & $8(26 \%)$ & 0.992 \\
\hline Hypertension & $125(48 \%)$ & $108(47 \%)$ & 17 (56\%) & 0.340 \\
\hline Lung disease & $84(33 \%)$ & $72(31 \%)$ & $12(40 \%)$ & 0.357 \\
\hline Obesity & $29(11 \%)$ & $26(11 \%)$ & $3(10 \%)$ & 0.810 \\
\hline Immunodeficiency & $23(9 \%)$ & $18(8 \%)$ & $5(17 \%)$ & 0.122 \\
\hline Neurological disorder & $15(6 \%)$ & $13(6 \%)$ & $2(6 \%)$ & 0.832 \\
\hline Laboratory Results ${ }^{\mathrm{b}}$ & $n=253$ & $n=223$ & $n=30$ & \\
\hline Influenza virus (A or $B$ ) & 47 (19\%) & $43(19 \%)$ & $4(13 \%)$ & 0.464 \\
\hline Parainfluenza virus (1, 2 or 3 ) & $13(5 \%)$ & $12(5 \%)$ & $1(3 \%)$ & 0.653 \\
\hline Coronavirus (OC43, HKU1, NL63, 229E) & $14(6 \%)$ & $12(5 \%)$ & $2(6 \%)$ & 0.750 \\
\hline Human metapneumovirus & $20(8 \%)$ & $18(8 \%)$ & $2(6 \%)$ & 0.754 \\
\hline Respiratory syncytial virus & $8(3 \%)$ & $8(3 \%)$ & $\mathrm{n} / \mathrm{a}$ & \\
\hline Rhinovirus & $10(4 \%)$ & $9(4 \%)$ & $1(3 \%)$ & 0.870 \\
\hline Any viral pathogen ${ }^{c}$ & $113(45 \%)$ & $103(46 \%)$ & $10(33 \%)$ & 0.410 \\
\hline
\end{tabular}

${ }^{\mathrm{a}}$ Six SARI patients missing data on clinical suspicion of pneumonia; $n=252$

${ }^{\mathrm{b}}$ Five SARI patients did not have laboratory results; $n=253$

'Three patients were co-infected; one with coronavirus HKU1/rhinovirus, one with coronavirus HKU1/human metapneumovirus and one with coronavirus 229E/rhinovirus

underlying respiratory and circulatory. All deaths were of natural causes.

\section{Predictors of 30-day mortality}

In bivariate analysis, only two factors were statistically significant in predicting 30-day mortality: admission to the ICU (OR 5.7; 95\% CI 2.4-13.4) and age $\geq 65$ years (OR 2.8; 95\% CI 1.2-6.5) (Table 2). In multivariate analyses, ICU admission (OR 7.4; 95\% CI 3.0-17.9) and age $\geq$ 65 years (OR 4.0; 95\% CI 1.6-9.9) remained independent significant predictors of 30-day mortality. Kaplan-Meier curves of 30-day mortality by ICU admission and age group are shown in Figs. 1 and 2. ICU admission and age $\geq 65$ years were associated with poorer survival at 30 days post admission (log-Rank test: $\mathrm{p} 0.01$ and $p<0.0001$, respectively).

\section{Discussion}

In this Southern Arizona population, mortality among adult persons hospitalized with SARI was $9 \%$ and increased to $12 \%$ when a 30 -day post-hospitalization window was considered. This demonstrated that the use of electronic vital records increased SARI-associated mortality estimates among adults by $30 \%$ by capturing 
Table 2 Bivariate and multivariate analyses for predictors of mortality

\begin{tabular}{|c|c|c|c|c|}
\hline \multirow[b]{2}{*}{ Factor } & \multicolumn{2}{|l|}{ Bivariate analysis } & \multicolumn{2}{|l|}{ Multivariate analysis } \\
\hline & Odds Ratio $(95 \% \mathrm{Cl})$ & $p$-value & Odds Ratio $(95 \% \mathrm{Cl})$ & $p$-value \\
\hline Age $\geq 65$ years & $2.8(1.2-6.5)$ & 0.01 & $4.0(1.6-9.9)$ & 0.002 \\
\hline Admission to intensive care unit & $5.7(2.4-13.4)$ & $<0.0001$ & $7.4(3.0-17.9)$ & $<0.0001$ \\
\hline$\geq 1$ comorbidity & $2.4(0.7-8.2)$ & 0.16 & - & - \\
\hline Hypertension & $1.4(0.6-3.1)$ & 0.34 & - & - \\
\hline Chronic obstructive pulmonary disease & $1.4(0.7-3.1)$ & 0.35 & - & - \\
\hline Duration of hospital stay & $1.0(0.9-1.1)$ & 0.48 & - & - \\
\hline Antivirals given & $0.6(0.1-2.8)$ & 0.54 & - & - \\
\hline Sex (female) & $1.2(0.5-2.5)$ & 0.67 & - & - \\
\hline \multicolumn{5}{|l|}{ Race/Ethnicity ${ }^{a}$} \\
\hline Asian & $2.6(0.3-26.4)$ & 0.53 & - & - \\
\hline African American & $0.5(0.6-4.1)$ & 0.42 & - & - \\
\hline Hispanic & $1.0(0.5-2.3)$ & 0.91 & - & - \\
\hline Native American & $1.5(0.2-14.1)$ & 0.69 & - & - \\
\hline Duration of hospital stay & $1.0(0.9-1.1)$ & 0.48 & - & - \\
\hline Obese & $0.8(0.2-3.0)$ & 0.81 & - & - \\
\hline
\end{tabular}

${ }^{\mathrm{a}}$ Referent group $=$ White, non-Hispanic

deaths occurring outside the hospital; when limited to persons $\geq 65$ years the increase was $47 \%$. Age $\geq 65$ years and admission to the ICU were significantly associated with mortality.

Given the lack of SARI studies in the United States, hospitalized adults with conditions such as communityacquired pneumonia (CAP) may be the best comparison group for SARI patients in this analysis. The 9\% inhospital mortality in this study was similar to one study of adults hospitalized with CAP in San Antonio, Texas (10\%) but higher than in-hospital mortality in the Etiology of Pneumonia in the Community (EPIC) study $(2 \%)[17,18]$. However, there are differences in the case

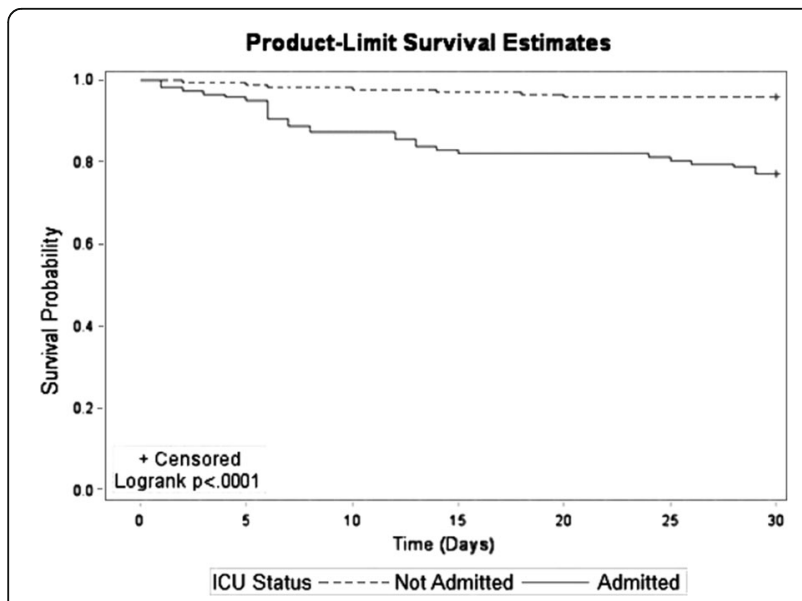

Fig. 1 Kaplan Meier Estimate for 30-day survival post-hospital admission by age group definition between SARI and CAP that may affect direct comparisons. Additionally, in our surveillance, a larger proportion of SARI patients were admitted to the ICU ( $37 \%$ vs. $21 \%)$ and aged $\geq 65$ years $(56 \%$ vs. $36 \%)$ when compared to the proportions of CAP patients in the EPIC study, and this could have contributed to the higher observed mortality.

Globally, there are more SARI data for comparison. In-hospital mortality was $6 \%$ among adult SARI patients in eight African countries from 2009 to 2012 and 8\% in a South African population with a high prevalence of HIV from 2012 to 2013 [19, 20]. In a study of SARI patients $\geq 18$ years in Bogotá, Colombia, Remolina et al.

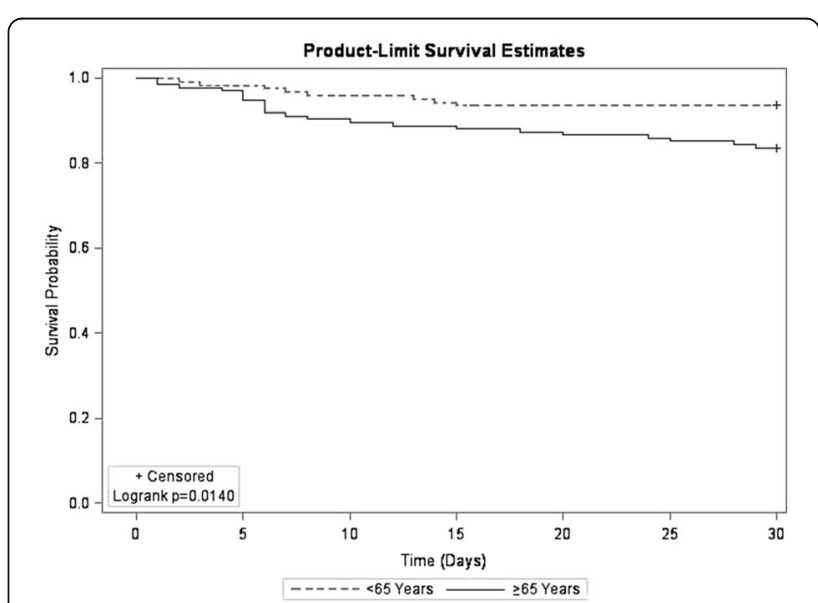

Fig. 2 Kaplan Meier Estimate for 30-day survival post-hospital admission by ICU status 
reported higher in-hospital mortality of $15 \%$ [3]. One study of adult SARI patients in central China reported an overall mortality of $4 \%$ up to 30 -days post discharge [21]. Again, the higher in-hospital mortality in our surveillance compared to other global SARI studies is not surprising given the 37\% ICU admission and high median age of Arizona SARI patients.

The primary goal of this analysis was to better assess mortality in SARI patients by capturing additional deaths occurring soon after hospital discharge. Among adults $\geq$ 65 years hospitalized with CAP, previous studies have shown that nearly half of those who died did so after discharge [22]. Among Canadian adults hospitalized with CAP, Johnstone et al. reported a mortality of $12 \%$ at 30 days and $28 \%$ at one year follow-up [23]. A known limitation of the Arizona SARI surveillance program was that only in-hospital deaths were captured, and deaths occurring in patients discharged to hospices, nursing homes or private residences were not included. By using vital records, seven additional deaths occurring between hospital discharge and 30-days post admission were captured, supporting claims that mortality estimates for SARI were previously underestimated in Southern Arizona [11]. Although there is no gold standard for attributing cause of death to SARI, mortality studies that use vital records and administrative data to capture in-hospital and post discharge mortality may provide guidance for a more accurate way to estimate the proportion of deaths attributable to SARI. While hospital discharge practices vary globally, cross-country comparison of mortality studies may benefit from use of a 30-day post admission timeframe.

The independent risk factors for mortality that were identified in this analysis, age $\geq 65$ years and ICU admission, are consistent with those found in a limited number of studies reporting risk factors for mortality in all SARI patients [20, 24]. Of 1790 adult and adolescent SARI patients in China, age $\geq 65$ years was a significant risk factor for severe SARI outcomes (ICU admission or death) [21]. Among SARI patients aged $\geq 5$ years in a South African population with high-HIV-prevalence, Cohen et al. found that ICU admission was a significant risk factor in bivariate analysis while increasing age was an independent risk factor in multivariate analysis [20]. As with previous studies assessing the effect of ICU admission on patient outcome, it is important to consider that our findings are likely subject to confounding by indication. This type of bias arises because patients with more severe illness are more likely to be admitted to the ICU, and more likely to die. Although we expected higher mortality given the 37\% ICU admission in our analysis, one study of U.S. Medicare beneficiaries found lower 30-day mortality among patients with discretionary admission to the ICU after adjusting for distance to hospitals with high ICU admission rates for pneumonia [25].
Nineteen percent $(47 / 253)$ of SARI patients tested positive for influenza viruses in this analysis. We can make some comparisons to other studies with the caveat that varying populations, years studied and enrollment methods may affect the findings. The proportion of influenza-positive SARI cases in our study was higher than reported in Kenya (10\%) [26] and China (16\%) [21], within range of eight African countries (5\% in Tanzania to $26 \%$ in Madagascar) [19] and slightly lower than New Zealand (23\%) [27]. In our surveillance system, the percent of SARI cases positive for an influenza virus varied by year from $5.6 \%$ to $20 \%$ and likely reflects the influenza activity of the dominant virus that year [11].

This analysis had several strengths. First, the inclusion of several seasons of surveillance data provided a more robust understanding of SARI and SARI-associated mortality in this region and showed consistent mortality across the seasons included in this analysis. Second, the geographic location of the surveillance program in the border region allowed for enrollment of a larger proportion of minority populations such as Hispanics and Native Americans, which may provide a better understanding of the burden of SARI in these populations. Lastly, this analysis benefited from the standardized data collection methods of SARI surveillance in conjunction with electronically available state death certificate data. The result was a surveillance database with a high level of detail on risk factors, clinical diagnoses and outcomes for each patient, but particularly for patients who died and had death certificate data.

This surveillance system has several limitations that may have affected the results of this analysis. First, the exclusion of patients from one sentinel hospital contributed to the relatively small sample size which may have limited the ability to detect potential risk factors of mortality, such as cardiovascular, renal, and neurological disease which have been shown by others to contribute to poor survival of patients with influenza and pneumonia in this population [28]. Second, exclusion of bacterial culture results and admitting chest $\mathrm{x}$-ray findings prevented assessment of factors such as chest $\mathrm{x}$-ray abnormalities and bacterial co-infections as risk factors for mortality. Third, the SARI patients included in this analysis may not be representative of the population of Southern Arizona where an estimated 22 million people crossed the northbound Arizona border in 2014 alone $[11,29]$. Since sentinel sites in closer proximity to the U.S.-Mexico border were not included, the results of this analysis may not represent the burden of disease in the highly mobile border population. By limiting surveillance to large referral hospitals, we suspect there may be bias for certain risk groups, such as adults $\geq 65$ years old, and more severe cases presenting later in the course of illness. The high frequency of ICU admission in our 
patients supports this hypothesis. Additionally, the sentinel sites used for this analysis are not major providers of pediatric care in Southern Arizona so a similar pediatric SARI mortality estimate remains unavailable in this population. Lastly, the linkage of death certificate data to SARI patients could have been more thorough had we searched death registries in nearby states and used unique identifiers such as social security number, had they been available.

\section{Conclusions}

Surveillance for SARI in this region of the United States that has a highly fluid border population identified persons at high risk for severe respiratory illness and death, and these data may help target prevention strategies. The use of vital records provided additional information on the frequency and cause of mortality in patients with SARI and should be considered for use in other mortality studies when possible. Medical practitioners should be aware of a residual risk for mortality in SARI patients shortly after hospital discharge, especially in those aged $\geq$ 65 years or admitted to ICU, and should enhance post-discharge monitoring of these patients.

\section{Abbreviations}

ADHS: Arizona Department of Health Services; BIDS: Border Infectious Disease Surveillance; CAP: Community-acquired pneumonia; CDC: Centers for Disease Control and Prevention; HMPV: Human metapneumovirus; ICD-10: International classification of disease, 10th revision; ICU: Intensive care unit; PCR: Polymerase chain reaction; RSV: Respiratory syncytial virus; SARI: Severe acute respiratory infection; WHO: World Health Organization

\section{Acknowledgements \\ We would like to thank the staff of St Mary's hospital especially Daniel Lewis, Shannon Mabalot, and Ami Bera; the staff of St Joseph Hospital especially Cynthia Kartchner and Monica Fontes; the staff of Sells Hospital especially Guadalupe Camarena and Mary Bell. We are thankful to BIDS program at CDC Division of Global Migration and Quarantine, US-Mexico Unit: Dr. Steve Waterman, Alba Phippard, Clelia Pezzi, and Sonia Montiel. We also gratefully acknowledge the support of the Arizona Department of Health Services especially Ken Komatsu, Shane Brady and Robert Guerrero.}

\section{Funding}

This surveillance was supported by the Epidemiology and Laboratory Capacity for Infectious Diseases cooperative agreement (Grant number: 3U50CK0004) from the U.S. Centers for Disease Control and Prevention.

\section{Availability of data and materials}

The datasets generated and/or analyzed during the current study are not publicly available due to confidentiality agreement at the Arizona Department of Health Services but are available from the corresponding author on reasonable request.

\section{Disclaimer}

The findings and conclusions in this report are those of the authors and do not necessarily represent the official position of the Centers for Disease Control and Prevention or the Arizona Department of Health Services.

\section{Authors' contributions}

SRB had full access to all data in the study and takes responsibility for the integrity of the data and the accuracy of the data analysis. ZW, SJO and MGC conceived and designed the study. $\mathrm{KH}$ contributed to acquisition of Arizona State Vital Records. SRB and ZW performed statistical analysis. SRB, ZW, KH, $\mathrm{EO}, \mathrm{KE}, \mathrm{SJO}$ and MGC contributed to the analysis and interpretation of the data. SRB drafted the manuscript. SRB, ZW, KH, EO, KE, SJO and MGC critically revised the manuscript for important intellectual content. All authors read and approved the final manuscript.

\section{Ethics approval and consent to participate}

The Arizona Department of Health Services' Human Subjects Review Board determined that severe acute respiratory infection surveillance was part of public health practice to enhance the surveillance for diseases of public health importance and exempt from review by the Institutional Review Board. Informed verbal consent was obtained from participants.

\section{Consent for publication}

Not applicable.

\section{Competing interests}

The authors declare that they have no competing interests.

\section{Publisher's Note}

Springer Nature remains neutral with regard to jurisdictional claims in published maps and institutional affiliations.

\section{Author details}

${ }^{1}$ Arizona Department of Health Services, Border Infectious Disease Surveillance Program, 400 West Congress, Suite 116, Tucson, AZ 85701, USA. ${ }^{2}$ Mel and Enid Zuckerman College of Public Health, University of Arizona, 1295 N Martin Ave, Tucson, AZ 85724, USA. ${ }^{3}$ Arizona Department of Health Services, Office of Infectious Disease Services, 150 N 18th Ave Phoenix, Phoenix, AZ 85007, USA. ${ }^{4}$ Influenza Division, Centers for Disease Control and Prevention, 1600 Clifton Road, Atlanta, GA 30329-4027, USA.

Received: 21 June 2017 Accepted: 31 January 2018 Published online: 12 February 2018

\section{References}

1. Kochanek KD, Murphy SL, Xu JQ, Tejada-Vera B. Deaths: final data for 2014. In: national vital statistics reports. National Center for Health Statistics. 2016;65(4)

2. Lee N, Choi KW, Chan PKS, Hui DSC, Lui GCY, Wong BCK, et al. Outcomes of adults hospitalised with severe influenza. Thorax. 2010;65:510-5.

3. Remolina YA, Ulloa MM, Vargas $H$, Viral Infection DL. In adults with severe acute respiratory infection in Colombia. PLoS One. 2015;10(11):1-13.

4. Elliot AJ, Fleming DM. Influenza and respiratory syncytial virus in the elderly. Expert Rev Vaccines. 2008;7(2):249-58.

5. Mertz D, Kim TH, Johnstone J, Lam P-P, Science M, Kuster SP, et al. Populations at risk for severe or complicated influenza illness: systematic review and meta-analysis. BMJ. 2013;347:f5061.

6. Rolfes MA, Foppa IM, Garg S, Flannery B, Brammer L, Singleton JA, et al. Estimated Influenza IIInesses. Hospitalizations, and Deaths Averted by Vaccination in the United States: Medical Visits; 2016. https://www.cdc.gov/ flu/about/disease/2015-16.htm. Accessed 01 Aug 2017

7. Centers for Disease Control and Prevention NC for HS. Compressed Mortality File 1999-2015 on CDC WONDER Online Database. CMF 19992015, Series 20, No 2U. 2016. http://wonder.cdc.gov/cmf-icd10.html. Accessed 31 July 2017.

8. Arizona Health Status and Vital Statistics 2015 Annual Report. Arizona Department of Health Services 2015. http://pub.azdhs.gov/health-stats/ report/ahs/ahs2015/index.php. Accessed 31 July 2017.

9. World Health Organization. Global Epidemiological Surveillance Standards for Influenza. 2014. http://www.who.int/influenza/resources/documents/ influenza_surveillance_manual/en. Accessed 05 Jan 2017.

10. World Health Organization. Operational Guidelines for Sentinel Severe Acute Respiratory Infection ( SARI ) Surveillance September 2014. 2014. http:// www.paho.org/hq/index.php?option=com_docman\&task=doc_view\&gid= 31162\&ltemid=1639\&lang=en. Accessed 08 Aug 2017. 
11. Wansaula Z, Olsen SJ, Casal M, Golenko C, Erhart LM, Kammerer P, et al. Surveillance for severe acute respiratory infections (SARI) in southern Arizona, 2010-2014. Influenza Other Respir Viruses. 2016; https://doi.org/10.1111/irv.12360.

12. Popowitch EB, $\mathrm{O}^{\prime}$ Neill SS, Miller MB. Comparison of the biofire filmarray RP, Genmark eSensor RVP, Luminex xTAG RVPv1, and Luminex xTAG RVP fast multiplex assays for detection of respiratory viruses. J Clin Microbiol. 2013;51(5):1528-33.

13. Li H, McCormac MA, Estes RW, Sefers SE, Dare RK, Chappell JD, et al. Simultaneous detection and high-throughput identification of a panel of RNA viruses causing respiratory tract infections. J Clin Microbiol. 2007:45(7):2105-9.

14. Thompson WW, Shay DK, Weintraub E, Brammer L, Cox N, Anderson LJ. Mortality associated with influenza and respiratory syncytial virus in the United States. JAMA. 2003:289(2):179-86.

15. Mortensen EM, Coley CM, Singer DE, Obrosky T, Scott MD, Kapoor WN. Causes of death for patients with community-acquired pneumonia: results from the pneumonia patient outcomes research team cohort study. Arch Intern Med. 2002;162:1059-64.

16. Centers for Medicare \& Medicaid Services 30-Day Mortality Measures. Specifications Manual for National Hospital Inpatient Quality Measures. 2012. 3.2: 7-11.

17. Peper JS, Dahl RE. Community-acquired pneumonia requiring hospitalization among U.S. adults. N Engl J Med. 2015;373(5):415-27.

18. Restrepo MI, Mortensen EM, Velez JA, Frei C, Anzueto A. A comparative study of community-acquired pneumonia patients admitted to the ward and the ICU. Chest. 2008;133(3):610-7.

19. McMorrow ML, Wemakoy EO, Tshilobo JK, Emukule GO, Mott JA, Njuguna H, et al. Severe acute respiratory illness deaths in sub-Saharan Africa and the role of influenza: a case-series from 8 countries. J Infect Dis. 2015;212(6):853-60.

20. Cohen C, Walaza S, Moyes J, Groome M, Tempia S, Pretorius M, et al. Epidemiology of severe acute respiratory illness (SARI) among adults and children aged $\geq 5$ years in a high HIV-prevalence setting, 2009-2012. PLoS One. 2015;10(2):e0117716.

21. Zheng J, Huo X, Huai $Y$, Xiao L, Jiang H, Klena J. Epidemiology, seasonality and treatment of hospitalized adults and adolescents with influenza in Jingzhou, China, 2010-2012. PLoS One. 2016;11(3):e0150713.

22. Metersky ML, Waterer G, Nsa W, Bratzler DW. Predictors of in-hospital vs postdischarge mortality in pneumonia. Chest. 2012;142(2):476-81.

23. Johnstone J, Eurich DT, Majumdar SR, Jin Y, Marrie TJ. Long-term morbidity and mortality after hospitalization with community-acquired pneumonia. Medicine (Baltimore). 2008;87(6):329-34.

24. Peng Z, Feng L, Carolyn GM, Wang K, Zhu G, Zhang Y, et al. Characterizing the epidemiology, virology, and clinical features of influenza in China's first severe acute respiratory infection sentinel surveillance system, February 2011-October 2013. BMC Infect Dis. 2015;15:143.

25. Valley TS, Sjoding MW, Ryan AM, Iwashyna TJ, Cooke CR. Association of Intensive Care Unit Admission with Mortality among Older Patients with Pneumonia. JAMA. 2015:314(12):1272-9.

26. Katz MA, Muthoka P, Emukule GO, Kalani R, Njuguna H, Waiboci LW, et al. Results from the first six years of national sentinel surveillance for influenza in Kenya, July 2007-June 2013. PLoS One. 2014;9(6):e98615.

27. Huang QS, Baker M, McArthur C, Roberts S, Williamson D, Grant C, et al. Implementing hospital-based surveillance for severe acute respiratory infections caused by influenza and other respiratory pathogens in New Zealand. West Pacific Surveill Response J. 2014:5(2):23-30.

28. Quandelacy TM, Viboud C, Charu V, Lipsitch M, Goldstein E. Age- and sex-related risk factors for influenza-associated mortality in the United States between 1997 - 2007. Am J Epidemiol. 2014;179(2):156-67.

29. Bureau of Transportation Statistics. Arizona Border Crossing/Entry Data 2014 2017. https://transborder.bts.gov/programs/international/transborder/TBDR_ BC/TBDR BCQ.html. Accessed 24 Mar 2017

\section{Submit your next manuscript to BioMed Central and we will help you at every step:}

- We accept pre-submission inquiries

- Our selector tool helps you to find the most relevant journal

- We provide round the clock customer support

- Convenient online submission

- Thorough peer review

- Inclusion in PubMed and all major indexing services

- Maximum visibility for your research

Submit your manuscript at www.biomedcentral.com/submit

) Biomed Central 